\title{
Central and extrapontine myelinolysis affecting the brain and spinal cord. An unusual presentation of pancreatic encephalopathy
}

\section{Alejandro Hornik, Federico J. Rodriguez Porcel, Caroline Agha, Murray Flaster, Sarkis Morales Vidal, Michael J. Schneck, John Lee and José Biller*}

Loyola University Medical Center, Chicago, IL, USA

\section{Edited by:}

Osvaldo Fustinoni, Universidad de

Buenos Aires, Argentina

Reviewed by:

Maria Zurru, Hospital Italiano,

Argentina

Luciano A. Sposato, INECO

Foundation, Argentina

Emilia Gatto, Sanatorio de la Trinidad

Mitre, Argentina

*Correspondence:

José Biller, Department of Neurology,

Stritch School of Medicine, Loyola

University Chicago, 2160 S. 1st

Avenue, Bldg. 105, Room 2700,

Maywood, IL 60153, USA.

e-mail: jbiller@lumc.edu
Pancreatic encephalopathy refers to a gamut of neuropsychiatric symptoms complicating acute pancreatitis. Osmotic myelinolysis is a known complication of pancreatic encephalopathy. We evaluated a 58-year-old woman with pancreatic encephalopathy associated to pontine and extrapontine myelinolysis involving the brain and spinal cord. To our knowledge, this is the first clinic pathological case report of pancreatic encephalopathy involving the spinal cord.

Keywords: pancreatic encephalopathy, osmotic myelinolysis, extrapontine myelinolysis, pontine myelinolysis, leukoencephalopathy
A 58-year-old right handed woman was brought to the emergency room after being found on the floor by her daughter. Fifteen days prior to admission she was admitted to an outside hospital for an ampulla of Vater adenoma resection and pancreatic duct stenting. An endoscopic retrograde cholangiopancreatography (ERCP) was complicated by acute pancreatitis (serum lipase $>5000$ units/L) due to stent migration. The stent was removed 2 days following the procedure. No electrolyte derangements were observed during her hospitalization. She remained hospitalized for another week and was reported to have "manipulative behavior." It was also noted that 1 week prior to admission to our hospital (1 week post-surgery), she was diagnosed with depression and attention deficit disorder and was started on methylphenidate and bupropion. Her family stated she had become "less conversational, sleepy, and odd" and referred to a psychiatrist.

Medical history was remarkable for a chronic postprandial abdominal pain, hypertension, and a large calcified pelvic mass assumed to be a leiomyoma. Outpatient medications included lisinopril, tramadol, sustained release bupropion, and methylphenidate. Upon admission to our hospital, she complained of persistent abdominal pain and somnolence.

On examination, there was an apical systolic murmur, diffuse abdominal discomfort to palpation, normal bowel sounds, and no peritoneal signs.

Initial blood work showed a hemoglobin of $13.1 \mathrm{~g} / \mathrm{dL}$, white blood cell count (WBC) of $16.6 \mathrm{~K} / \mathrm{UL}$ (lymphocytes $4 \%$, monocytes $10 \%$, granulocytes $85 \%$ ), platelets $324 \mathrm{~K} / \mathrm{UL}$. Basic metabolic profile (BMP), liver enzymes, serum albumin, and billirubin were unremarkable. Serum amylase was $92 \mathrm{U} / \mathrm{L}$ and serum lipase was $31 \mathrm{U} / \mathrm{L}$. Venous ammonia, urine drug screen, thyroid function tests, and troponins were unremarkable. Urinalysis showed moderate amounts of ketones and elevated urobilinogen. Activated partial thromboplastin time (aPTT) and International Normalized Ratio (INR) were normal. Cranial computed tomography (CCT) was unremarkable. Computed tomography (CT) of the chest, abdomen, and pelvis showed a $6 \mathrm{~mm}$ right hilar node, inflammatory changes of the pancreas consistent with pancreatitis, peripancreatic air, and abscess formation. There was also a $4 \mathrm{~cm} \times 7 \mathrm{~cm} \times 4 \mathrm{~cm}$ well defined soft tissue mass lesion with central calcification in the left lower quadrant of the abdomen (Figure 1). CT findings were remarkably improved compared to CT of the abdomen taken 3 weeks prior. General surgery consultant recommended intravenous meropenem. Transthoracic echocardiography (TTE) showed a left ventricular ejection fraction of $60 \%$, moderate to severe aortic stenosis, and infero-lateral hypokinesis.

Twelve hours following admission to our hospital, she became unarousable and was transferred to the Medical Intensive Care Unit (ICU). Neurology consultation was requested. Examination showed a comatose patient with a normal breathing pattern. She had symmetric facial grimacing to sternal rubbing. Pupils were $3 \mathrm{~mm}$ symmetric and reactive. Oculocephalic reflexes were normal. She had a flaccid areflexic tetraparesis, No extensor or flexor posturing were noted.

Magnetic resonance imaging (MRI) of the brain showed multiple areas of confluent restricted diffusion involving the splenium 


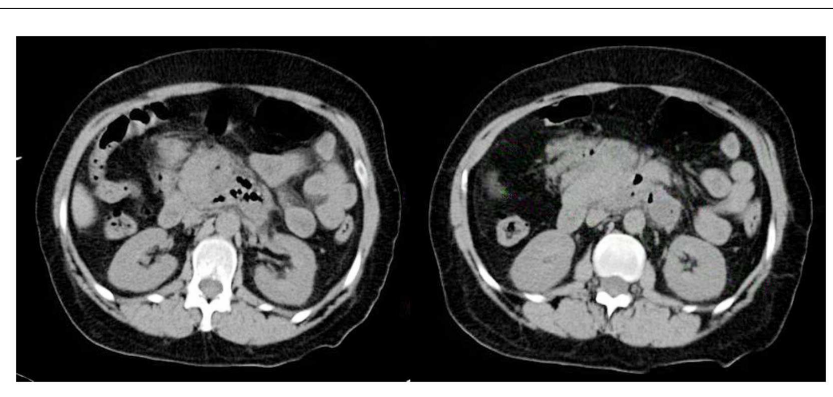

FIGURE 1 | CT of the abdomen shows an abnormal, poorly defined collection $(6.9 \mathrm{~cm} \times 4.7 \mathrm{~cm})$ of gas and inflammatory changes in the region of the head and uncinate process of the pancreas and dorsal and inferior to the body of the pancreas. A more focal

$(2.1 \mathrm{~cm} \times 1.9 \mathrm{~cm})$ fluid density along the posterior left aspect is noted.

of the corpus callosum, right medial occipital white matter, right brachium pontis, bilateral dorsal pons, and right cerebral peduncle. These regions were associated with non-enhancing hyperintense $\mathrm{T} 2$ and fluid attenuated inversion recovery (FLAIR) sequence without mass effect (Figure 2).

A lumbar puncture (LP) showed an opening pressure of $19 \mathrm{~cm}$ of CSF. There were 176 RBC, $280 \mathrm{WBC}$ (74\% segmented, $2 \%$ lymphocytes, $10 \%$ monocytes), glucose content of $49 \mathrm{mg} / \mathrm{dL}$ (30\% of serum glucose), and a protein content of $106 \mathrm{mg} / \mathrm{dL}$. Smears, bacterial, viral, and fungal cultures, polymerase chain reaction (PCR) for Herpes Simplex Virus (HSV) type 1 and 2, flow cytometry, and cytology were negative.

The patient received intravenous Vancomycin 1 gram every $12 \mathrm{~h}$, cefepime $2 \mathrm{~g}$ every $12 \mathrm{~h}$, ampicillin $2 \mathrm{~g}$ every $4 \mathrm{~h}$, metronidazole $500 \mathrm{mg}$ every $6 \mathrm{~h}$, and acyclovir $10 \mathrm{mg} / \mathrm{kg}$ every $12 \mathrm{~h}$. Thirty-six hours after admission the patient exhibited anisocoria; the right pupil was $4 \mathrm{~mm}$, sluggish and reactive; the left pupil was $2 \mathrm{~mm}$ and reactive. After elective intubation for airway protection, a repeat CCT was unchanged.

Repeat LP showed 106 RBC, 3 WBC (4\% segmented, 86\% lymphocytic, 10\% macrophages), glucose content of 68 (45\% of serum), and a protein content of 180 . Smears, cultures, flow cytometry, and cytology again were negative. PCR for Epstein Bar virus (EBV), JC virus, cytomegalovirus (CMV), and cryptococal antigen were negative. Oligoclonal bands and myelin basic protein were negative.

Serial blood cultures failed to grow any bacteria. Serum human immunodeficiency virus (HIV) antibodies and HIV PCR analysis were negative. Serum paraneoplastic antibody panel (anti $\mathrm{Hu}$, Ma1, Ma2, Yo, Ri, Car, Lems, CV2, Zix4, VGKC, Amphiphysin, G-ACHR), antiphospholipid antibody panel (beta 2 glycoprotein IgG, IgM, cardiolipin IgG, IgM), thyroid panel including thyroid stimulating hormone (TSH), free T4, T3, and thyroperoxidase (TPI) antibodies were all unremarkable.

The patient was transferred to the Neuroscience ICU (NICU). All antimicrobials were discontinued after 7 days of treatment. Repeat CT of the abdomen showed improvement of the pancreatic and peripancreatic abnormalities. Repeat MRI of the brain showed increased size, on diffusion weighted images, and T2/FLAIR, of the lesions located in the splenium, posterior white matter, midbrain, pons, and cerebellum. These lesions had faint enhancement following gadolinium administration (Figure 3). MRI of the cervical spine showed multiple non-contiguous spinal cord expansive lesions from the cervico-medullary junction to the level of the sixth cervical vertebra (Figure 4).

Electroencephalography (EEG) showed moderate to severe diffuse background slowing consistent with a moderate to severe encephalopathy with a possible focal epileptiform activity arising from the right frontal region. The patient received $1500 \mathrm{mg}$ of IV levetiracetam followed by $750 \mathrm{mg}$ every $12 \mathrm{~h}$ with resolution of the ictal electrographic phenomenon. Somatosensory evoked potentials (SSEPs) of the upper and lower extremities were uninterpretable due to technical difficulties. Visual evoked potentials (VEP) showed integrity of the visual pathways.

A right burr hole stereotactic brain biopsy of the splenium and right occipital lobe showed reactive gliosis and numerous macrophages. Specific stains for differentiation CD20 (B-cell) and CD3 (T-cell) showed no prominent lymphocytic infiltrates. Luxol Fast Blue and Bielschowsky stains showed evidence of demyelination, with relative preservation of axons. Differential diagnosis at this point included pontine and extrapontine myelinolysis (EPM). Despite the scant presence of perivascular lymphocytic inflammatory infiltrates, a diagnosis of possible acute disseminated encephalomyelitis (ADEM) was also entertained.

The patient received $1500 \mathrm{mg}$ of IV methylprednisolone daily for 5 days followed by plasmapheresis for a total of five plasma exchanges. However, she had no clinical improvement. The patient remained hospitalized in critical condition for 3 weeks. Repeat brain and cervical spine MRIs showed no improvement. Hospital course was complicated by ventilator associated pneumonia and hypotension requiring vasopressors. Based on advanced directives, the family decided to discontinue vital support 5 weeks after initial admission to our hospital. She suffered a respiratory arrest 4 days after extubation resulting in her death.

\section{AUTOPSY FINDINGS}

Macroscopic analysis showed thin translucent leptomeninges without subarachnoid hemorrhage. There were multiple distinct white matter lesions including the right occipital lobe, splenium, and right middle cerebellar peduncle extending into the central pons. These multiple white matter areas contained numerous macrophages with focal cavitation containing scattered perivascular and parenchymal T cells (CD3) and B-cells (CD20). The neurofilament stains revealed preservation of the axons at the periphery of the lesions, but there was axonal loss in the middle of the cavitary portions of some of the lesions. Luxol Fast Blue myelin stains showed areas of demyelination including portions of the medullary medial lemniscus. Also noted were hyalinized barrelshaped vessels in the parietal and occipital cerebral cortical white matter as well as in the basal ganglia and amygdale consistent with mild arteriolar sclerosis. Focal gliosis and microglial proliferation in the thalamus was noted. A simian virus antibody 40 (SV40) stain for progressive multifocal leukoencephalopathy (polyoma virus) was negative. In summary, there were multiple cavitated pontine and extrapontine myelinolytic type lesions with extensive macrophages, focal cavitation, and scant inflammatory infiltrates 

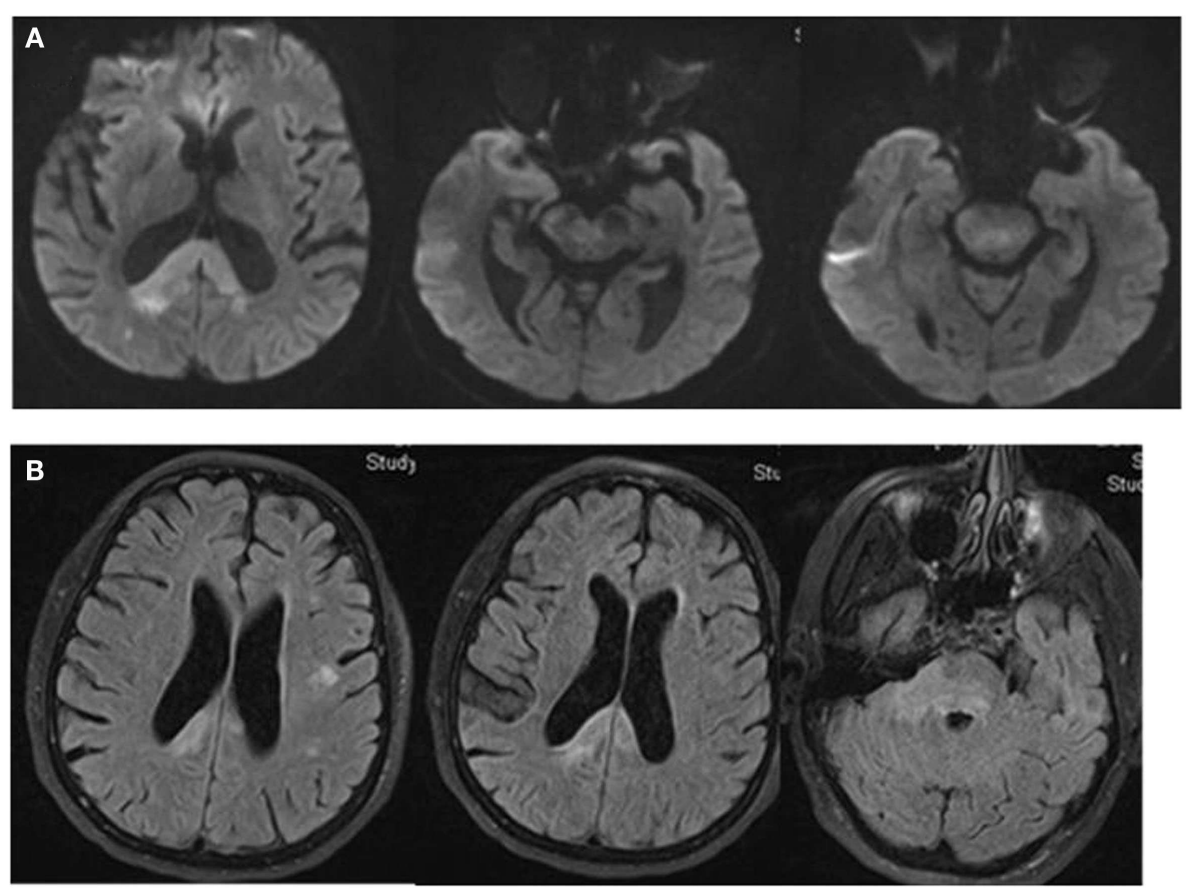

FIGURE 2 | DW-MRI of the brain shows multiple areas of confluent restricted diffusion affecting the splenium of the corpus callosum, right medial occipital white matter, right brachium pontis, bilateral dorsal pons, and right cerebral peduncle (A). These areas are hyperintense on Fluid Attenuated Inversion Recovery (FLAIR) sequence. There is no evidence of mass effect (B)

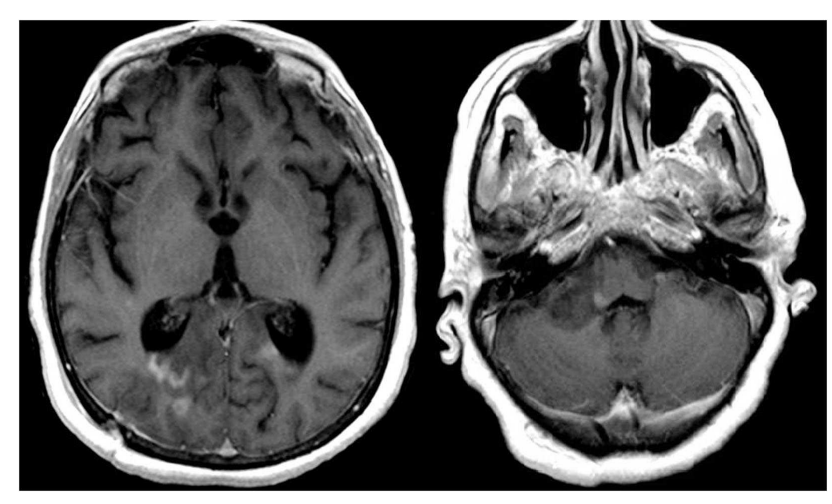

FIGURE 3 |T1W-MRI with contrast shows lesions located in the splenium, pons, and cerebellum with minimal contrast enhancement.

in the setting of peripheral pancreatic autolytic changes and peripancreatic fat necrosis and chronic inflammation consistent with pancreatic encephalopathy (Figures 5 and 6).

\section{DISCUSSION}

The osmotic myelinolytic syndromes combine signs and symptoms of central nervous system (CNS) myelinolysis. These disorders either affect the pons resulting in central pontine myelinolysis (CPM), or other CNS resulting in EPM.

Typical symptoms of CPM are biphasic (Adams et al., 1959). Initially, presenting with encephalopathy or seizures due to

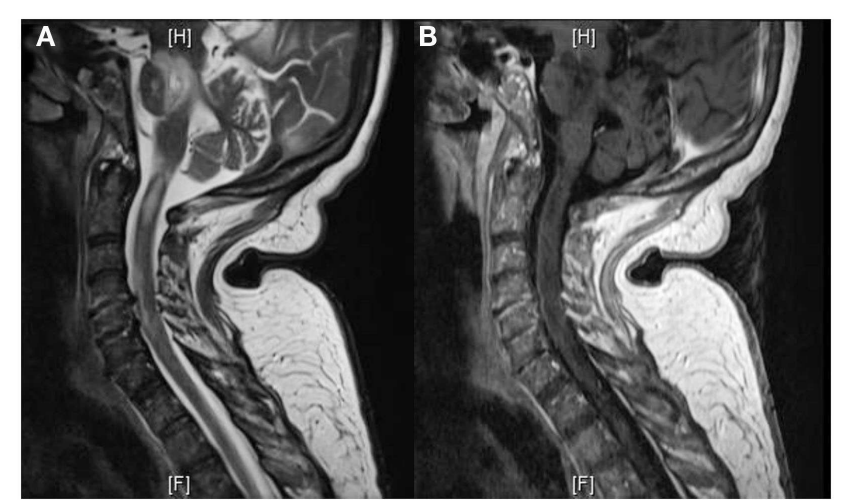

FIGURE 4 |T2W-MRI of the cervical spine shows multiple non-contiguous expansive lesions from the cervico-medullary junction to the level of the sixth cervical vertebra (A). T1W-MRI with contrast demonstrates patchy enhancement (B).

hyponatremia. As normonatremia is established, patients recover to subsequently deteriorate days later with a flaccid and then spastic quadriparesis, dysphagia, and dysarthria. If the myelinolytic process extends to the tegmentum, pupillary, and oculomotor abnormalities are noted.

Extrapontine myelinolysis may affect multiple areas within the CNS (Table 1). CPM and EPM may occur in isolation or combined (Gotch and Calmant, 1987) as in our patient. The coexistence of CPM and EPM may account for a protean clinical picture, often 
preceded by a variety of psychiatric manifestations. The underlying pathology of these lesions is characterized by degeneration and loss of oligodendrocytes with preservation of axons. Lesions are sharply demarcated. During the active phase of the disease, they contain sheets of lipid-laden macrophages and large number of reactive astrocytes. Infiltration by lymphocytes is sparse or absent (Love, 2006). A variety of potential etiologies have been identified (Table 2; Tomlinson et al., 1976; Martin, 2004). Patients with osmotic myelinolysis also have a high prevalence of concomitant hypokalemia (Lohr, 1994). Myelinolysis is best appreciated on MRI, presenting with non-enhancing hyperintense lesions on T2 and hypointense lesions on T1.

Pancreatic encephalopathy (Rothermich and Von Haam, 1941), refers to a variety of neuropsychiatric symptoms complicating acute pancreatitis (Jacewicz and Marino, 2007). Encephalopathy has been reported in 9-35\% of subjects suffering acute pancreatitis without history of alcoholism (de Falco et al., 1980). Neurological manifestations related to acute pancreatitis are multifactorial

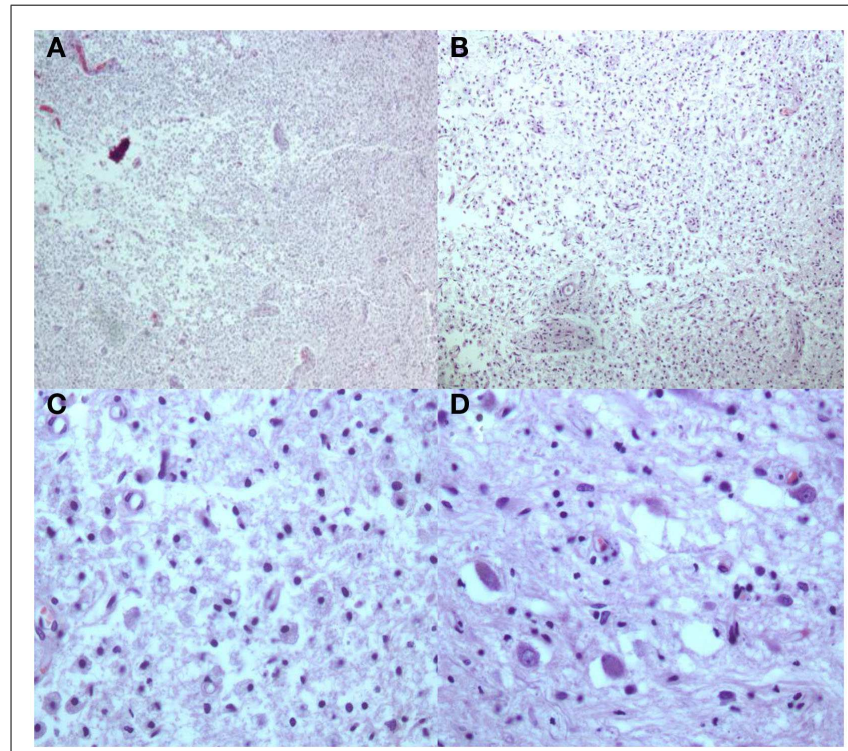

FIGURE 5 | H and E stains [(A,B) Low Power; (C) High Power] Multiple white matter areas containing myelin loss and numerous

macrophages with focal cavitation. Adjacent to areas of myelin loss there is relative preservation of neurons [(D) High Power]. and may result from hypocalcemia, hypomagnesemia, low thiamine levels, or osmotic myelinolysis. Symptoms due to osmotic myelinolysis include but are not limited to fluctuating mental status, disorientation, confusion, dysarthria, hallucinations, delirium, akinetic mutism, seizures, and coma (Sharf and Bental, 1971). Symptoms usually present 2-5 days after onset (Chan et al., 2003), although new neurological symptoms have been reported more than 1 month after onset of pancreatitis (Ding et al., 2004). CNS pathological changes include patchy myelin pallor (Vogel, 1951), CPM, EPM (Sherins and Verity, 1968), acute hemorrhagic leukoencephalitis (Chan et al., 2003), and fat embolism (Bhalla et al., 2003). Vogel first described scattered foci of intense demyelinization in cases of pancreatic encephalopathy. He was able

\section{Table 1 | CNS lesions on EPM.}

\section{Cerebellum}

Lateral geniculate body

External capsule

Extreme capsule

Hippocampus

Putamen

Subcortical areas

Thalamus

Caudate nucleus

Claustrum

Internal capsule

Midbrain

Internal medullary lamellae

Mamillary bodies

Medulla oblongata

\section{Table 2 | Disorders associated with myelinolysis.}

\section{Alcoholism}

Malnutrition

Prolonged diuretic use

Psychogenic polydipsia

Burns

Post liver transplant

Post pituitary surgery

Post urological/gynecological surgery (s/p glycine infusion)

Pancreatitis

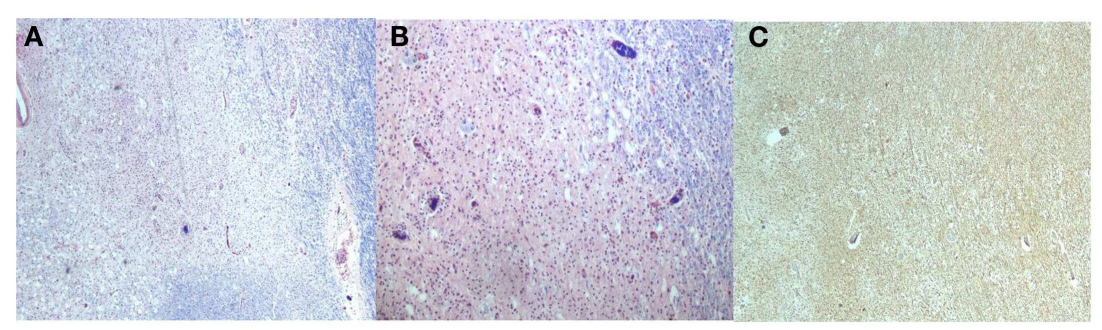

FIGURE 6 | Luxol Fast Blue stains [(A,B) Low power] shows areas with loss of myelin and increased macrophages. The neurofilament stain [(C), Low power] with preservation of the axons primarily at the periphery of the lesions. 
to demonstrate similar pathological effect in animals by injecting lipase into the CNS (Vogel, 1951). Other putative physiopathologic explanations have been postulated including pancreatin activation, cytokines such as tumor necrosis factor alpha (TNF- $\alpha$ ), Interleukin 1(IL 1). These pro-inflammatory markers and pancreatic enzymes were accounted to increase blood brain barrier permeability, causing vasogenic edema, myelinolysis, inflammatory activation, electrolytic disturbances, and hyperosmolarity due to osmotic diuresis (Zhang, 2007). Animal models of acute pancreatitis have subsequently proven the effect of the mentioned markers (Farkas et al., 1998). Differential diagnosis of altered mental status among patients with pancreatitis is extensive. Pancreatic encephalopathy should only be considered after more likely possibilities including ischemia, uremia, hypoxemia, electrolyte abnormalities, thiamine deficiency, have been excluded (Jacewicz and Marino, 2007). CSF analysis on patients with pancreatic encephalopathy show high protein content, mild lymphocytosis, and lipase (Vogel, 1950; Estrada et al., 1979; Sjaastad et al., 1979).

There is no known treatment for the osmotic demyelinating syndrome regardless of etiology. In cases of pancreatic

\section{REFERENCES}

Adams, R. A., Victor, M., and Mancall, E. L. (1959). Central pontine myelinolysis: a hitherto undescribed disease occurring in alcoholics and malnourished patients. AMA Arch. Neurol. Psychiatry 81, 154-172.

Bhalla, A., Sachdev, A., Lehl, S. S., Singh, R., and D'Cruz, S. (2003). Cerebral fat embolism as a rare possible complication of traumatic pancreatitis. JOP 4, 155-157.

Chan, C., Fryer, J., Herkes, G., Prelog, K., and Harrington, T. (2003). Fatal brain stem event complicating acute pancreatitis. J. Clin. Neurosci. 10, 351-358.

de Falco, F. A., Sollazzo, D., and Campanella, G. (1980). Pancreatic encephalopathy. A case report with multifocal neurological signs. Acta Neurol. (Napoli) 2, 482-487.

Ding, X., Liu, C. A., Gong, J. P., and Li, S. W. (2004). Pancreatic encephalopathy in 24 patients with severe acute pancreatitis. HBPD INT 3, 608-611.

Estrada, R. V., Moreno, J., Martinez, E., Hernandez, M. C., Gilsanz, G., and Gilsanz, V. (1979). Pancreatic encephalopathy. Acta Neurol. Scand. 59, 135-139.

Farkas, G., Marton, J., Nagy, Z., Mándi, Y., Takács, T., Deli, M. A., and Abrahám, C. S. (1998). Experimental acute pancreatitis results in increased blood-brain barier permeability in the rat: a potential role for tumor necrosis factor and interleukin 6. Neurosci. Lett. 242, 147-150.
Gotch, A., and Calmant, H. U. (1987). Central pontine and extrapontine myelinolysis: a report of 58 cases. Clin. Neuropathol. 6, 262-270.

Jacewicz, M., and Marino, C. R. (2007). "Disease of the pancreas," in Interface of Neurology and Internal Medicine, 1st Edn, Chap. 37, ed. J. Biller (Philadelphia: Lippincott Williams \& Wilkins), 238-244.

Kumar, S., Fowler, M., Gonzalez-Toledo, E., and Jaffe, S. L. (2006). Central pontine myelinolysis, an update. Neurol. Res. 28, 360-366.

Lohr, J. W. (1994). Osmotic demyelination syndrome following correction of hyponatremia: association with hypokalemia. Am. J. Med. 96, 408-413.

Love, S. (2006). Demyelinating disease. J. Clin. Pathol. 59, 1151-1159.

Martin, R. J. (2004). Central pontine and extrapontine myelinolysis: the osmotic demyelination syndromes. J. Neurol. Neurosurg. Psychiatr. 75(Suppl. 3), iii22-iii28.

Oya, S., Tsutsumi, K., Ueki, K., and Kirino, T. (2001). Reinduction of hyponatraemia to treat central pontinemyelinolysis. Neurology 57, 1931-1932.

Pneumatikos, J., Frangides, C., Malizos, K., Tsagourias, M., and Nakos, G. (1998). Acute myelinolysis in the cervical spinal cord. J. Trauma 44, 562-564.

Qian, Z.-Y., Miao, Y., Liu, X.-L., and Du, J.-H. (2001). Clinical experience on the therapy of

encephalopathy, specific anti-enzymes, such as aprotinin (Trasylol ${ }^{\circledR}$ ), have been proposed. This is a low molecular weight polypeptide produced by the parotid glands and lung tissues. Aprotinin inhibits the action of trypsin, chymotrypsin, kallikrein, fibrinolysin, and other proteolytic enzymes (Sharf and Levy, 1976). Other proposed alternatives for the treatment of myelinolysis include reinduction of hyponatremia (Oya et al., 2001), recombinant human growth hormone (Qian et al., 2001), plasmapheresis, IVIG, and corticosteroids (Kumar et al., 2006).

\section{SUMMARY}

Our patient had pathology proven pancreatic encephalopathy affecting large areas of the white matter throughout brain, brainstem, and spinal cord. The cystic cavitating components of our patient's lesions most likely could be explained by direct proteolytic effect of pancreatic enzymes, including lipase. To our knowledge, there is only one other report of a patient presenting with osmotic myelinolysis affecting the spinal cord, but without accompanying and supporting autopsy pathology findings (Pneumatikos et al., 1998).

pancreatic encephalopathy. Zhongguo Xiandai Putong Waike Jinzhan 4, 99-100.

Rothermich, N., and Von Haam, E. (1941). Pancreatic encephalopathy. J. Clin. Endocrinol. 1, 872-881.

Sharf, B., and Levy, N. (1976). "Pancreatic encephalopathy," in Handbook of Clinical Neurology Metabolic and Deficiency Diseases of the Nervous System, eds P. J. Vinken, G. W. Bruyn, and H. Klawans (Amsterdam: North Holland), 449-458.

Sharf, N., and Bental, E. (1971). Pancreatic encephalopathy. J. Neurol. Neurosurg. Psychiatr. 34, 375-361.

Sherins, R. J., and Verity, M. A. (1968) Central pontine myelinolysis associated with acute hemorrhagic pancreatitis. J. Neurol. Neurosurg. Psychiatr. 31, 583-588.

Sjaastad, O., Gjessing, L., Ritland, S., Blichfeldt, P., and Sandnes, K. (1979). Chronic relapsing pancreatitis, encephalopathy with disturbance in consciousness and CSF amino acid aberration. J. Neurol. 220, 83-94.

Tomlinson, B. E., Pierides, A. M., and Bradley, W. G. (1976). Central pontinemyelinolysis. Two cases with associated electrolyte disturbance. Q. J. Med. 45, 373-386.

Vogel, F. (1951). Cerebral demyelination and focal visceral lesions in a case of acute hemorrhagic pancreatitis, with a consideration of possible role of circulating enzymes in the causation of the lesions. AMA Arch. Pathol. 52, 355-362.
Vogel, F. A. (1950). Demyelination induced experimentally by means of lipase. Fed. Proc. 9, 347.

Zhang, X. P. (2007). TianH. Pathogenesis of pancreatic encephalopathy in severe acute pancreatitis. HBPD INT 6, 134-140.

Conflict of Interest Statement: The authors declare that the research was conducted in the absence of any commercial or financial relationships that could be construed as a potential conflict of interest.

Received: 16 July 2012; paper pending published: 29 July 2012; accepted: 06 September 2012; published online: 01 October 2012.

Citation: Hornik A, Rodriguez Porcel FJ, Agha C, Flaster M, Morales Vidal $S$, Schneck MJ, Lee J and Biller J (2012) Central and extrapontine myelinolysis affecting the brain and spinal cord. An unusual presentation of pancreatic encephalopathy. Front. Neur. 3:135. doi: 10.3389/fneur.2012.00135

This article was submitted to Frontiers in Neurology Education, a specialty of Frontiers in Neurology.

Copyright (C) 2012 Hornik, Rodriguez Porcel, Agha, Flaster, Morales Vidal, Schneck, Lee and Biller. This is an openaccess article distributed under the terms of the Creative Commons Attribution License, which permits use, distribution and reproduction in other forums, provided the original authors and source are credited and subject to any copyright notices concerning any third-party graphics etc. 